\title{
Variation in the Phenotype of Photosensitive Cells Produced from Human Fibroblast Cell Lines
}

\author{
Toshiyuki Ishii ${ }^{1}$, Chengzhu Yin ${ }^{1}$, Yuko Seko ${ }^{2}$, \\ Akihiro Umezawa ${ }^{3}$ and Makoto Kaneda ${ }^{1}$ \\ ${ }^{1}$ Department of Physiology, Nippon Medical School, Tokyo, Japan \\ ${ }^{2}$ Visual Functions Section, Department of Rehabilitation for Sensory Functions, Research Institute, \\ National Rehabilitation Center for Persons with Disabilities, Saitama, Japan \\ ${ }^{3}$ Department of Reproductive Biology, Center for Regenerative Medicine, National Institute for Child Health and Development, Tokyo, Japan
}

Background: Photoreceptors differentiated from somatic cells are a useful tool for transplantation and drug screening. We previously showed that photosensitive cells are differentiated from human fibroblasts by direct reprogramming. In induced pluripotent stem (iPS) cells or embryonic stem (ES) cells, the properties of differentiated cells differ among the source of cell lines. However, whether or not the properties of the photosensitive cells produced by direct reprogramming are controlled by the origin of the cell line remains unknown.

Methods: We compared the morphological and physiological properties of photosensitive cells induced by two fibroblast cell lines.

Results: The differentiated cells had larger somas and more primary processes than the non-infected cells in both cell lines. The degree of morphological change was statistically different between the two cell lines. In addition, physiological responses to light differed between the two cell lines. An outward current (photoreceptor-like response) was observed in both cell lines, while an inward current (intrinsically photosensitive retinal ganglion cell-like response) was observed only in one cell line under light stimulation.

Conclusions: These results suggest that photosensitive cells produced from different cell lines by direct reprogramming might express different phenotypes. (J Nippon Med Sch 2018; 85: 110-116)

Key words: direct reprogramming, differentiation, human fibroblast, photoreceptors, intrinsically photosensitive retinal ganglion cells

\section{Introduction}

Retinitis pigmentosa, age-related macular degeneration, and cone dystrophy lead to a loss of photoreceptors and retinal pigment epithelium. One feasible therapy for retinal disease is regenerative medicine. Photoreceptors derived from embryonic stem (ES) cells ${ }^{1}$ have been implicated for use as a source of photoreceptor cells. The technology for producing retinal sheets from ES/induced pluripotent stem (iPS) cells by self-organogenesis ${ }^{2}$ is promising for retinal transplantation, and retinal pigment epithelium (RPE) derived from human ES cells has been transplanted for macular degeneration as a safety trial' ${ }^{3}$.
The induction of photosensitive cells by direct reprogramming has two advantages for the establishment of new pharmaceutical approaches. Firstly, working with human embryos raises ethical issues that prevent the widespread application of human fetal tissues and human ES cells. Secondly, the induction of photosensitive cells by direct reprogramming of fibroblasts takes two weeks, whereas the induction of photoreceptors from iPS cells takes about six months. Our previous studies have found that introducing the CRX, RAX, NEUROD, and OTX2 (CRNO) genes into human somatic cells by direct reprogramming induces photosensitive cell $\mathrm{s}^{4.5}$. Therefore,

Correspondence to Makoto Kaneda, Department of Physiology, Nippon Medical School, 1-1-5 Sendagi, Bunkyo-ku, Tokyo 1138602, Japan

E-mail: mkaneda@nms.ac.jp

Journal Website (http://www2.nms.ac.jp/jnms/) 
photosensitive cells induced by direct reprogramming can become a useful means of establishing new pharmacological therapies for patients with various intractable retinal diseases.

Increasing evidence has recently shown that the type of ES and iPS cell lines affect the ability of cells to differentiate into specific cells ${ }^{6-9}$. In addition, the phenotypes of iPS cell lines differ ${ }^{6}$. These findings support the notion that variations of the intrinsic properties among original cells are represented by the phenotypes of the differentiated cells produced from iPS cell lines. Therefore, how much phenotypic variation is represented among cell lines, considering that photosensitive cells can be produced by direct reprogramming as a platform, is important to assess for the development of new pharmacological approaches. However, whether the phenotypes of differentiated cells induced by direct reprogramming are affected by the source cell lines has not yet been examined. Therefore, the present study examined whether differences in fibroblast cell lines can affect the phenotypes of photosensitive cells induced by direct reprogramming. Our data supported the possibility that the selection of cell lines might affect the phenotypes of differentiated cells, and that new pharmacological approaches for intractable retinal diseases could be designed at the level of each individual patient.

\section{Materials and Methods}

\section{Cell Culture}

Two strains of cultured human dermal fibroblasts were used: HDF-a (Human Dermal Fibroblasts-adult) obtained from ScienCell Research Laboratories and NHDF (Normal Human Dermal Fibroblasts) obtained from PromoCell. These two kinds of fibroblasts were designated as Fib\#1 and Fib\#2, respectively. The cells were cultured in medium recommended by the manufactures (Fibroblast Medium and Fibroblast Growth Medium 2, respectively). All experiments handling human cells were carried out in line with the tenets of The Declaration of Helsinki.

\section{Preparation and Infection of Recombinant Retrovirus}

Four full-length transcription factors, CRX (RRID, CCDS: 12706.1), RAX (RRID, CCDS: 11972.1), NEUROD (RRID, CCDS: 2283.1), and OTX2 (RRID, CCDS: 41960.1), were amplified from cDNAs prepared from total RNAs from the adult retina (Clontech CA, USA) by PCR and cloned into the Xmnl-EcoRV sites of pENTR11 (Invitrogen). Each vector contained one transcription factor, and a mixture of vectors was used.

Preparation and infection of recombinant retroviruses were performed as previously reported ${ }^{4,5}$. The resulting pENTR11-transcription factors were recombined with pMXs-DEST by use of LR recombination reactions as instructed by the manufacturer (Invitrogen). The retroviral DNAs were transfected into 293FT cells, and 3 days later, the media were collected and concentrated. The human dermal fibroblasts were infected with this media containing retroviral vector particles. In the present study, the combination of CRNO genes $(C R X+R A X+N E U R O D+$ OTX2) was introduced into two fibroblast cell lines to induce photosensitive cells ${ }^{4}$. After retroviral infection, the media were replaced with the DMEM/F12/B27 medium (GIBCO) supplemented with $40 \mathrm{ng} / \mathrm{mL}$ bFGF (PEPRO$\mathrm{TECH}$ ), $20 \mathrm{ng} / \mathrm{mL}$ EGF (PEPROTECH), fibronectin (R\& D), and 1\% FBS (Hyclone). The retrovirus-infected cells were cultured at $37^{\circ} \mathrm{C}$ in a $\mathrm{CO}_{2}$ incubator $\left(95 \% \mathrm{O}_{2}-5 \%\right.$ $\mathrm{CO}_{2}$ ) for up to 14 days. We transfected retroviral eGFP under the same conditions to measure the efficiency of the infection. The frequency of eGFP-positive cells was 90-94\% of all cells at $48 \mathrm{~h}$ after infection.

\section{Patch Clamp Recording}

Experiments were carried out under non-blinded conditions. Cells cultured in the dark for longer than 8 hours were used, and experiments were conducted under a dim red light. For the light stimulation, a high pressure UV lamp (USH-102D, Ushio) was used as a light source. Diffuse, unpolarized blue light was generated through bandpass filters attached to a fluorescent emission system (BX-FLA, Olympus, Tokyo, Japan). The wavelength of the light for stimulation was $460-490 \mathrm{~nm}$. The duration and timing of the light stimulation was controlled by an electrically controlled shutter attached to a UV lamp box. The trigger signals to the electrically controlled shutter were given by commercially available software (pClamp 9) through AD/DA. The light intensity used for stimulation was $390 \mathrm{~W} / \mathrm{m}^{2}$. To activate the phototransduction cascade, 11-cis retinal (a gift from the vision research community, the National Eye Institute, National Institutes of Health) was added to the culture medium of human fibroblasts approximately $20 \mathrm{~min}$ before the electrical recording (final concentration: $37.5 \mu \mathrm{M}$ ). The importance of the addition of 11-cis retinal to evoke light responses was previously confirmed in studies from our group in human iris cells (unpublished observation). Electrical recordings were made in the whole-cell patch-clamp configuration. The recordings were performed in L-15 medium (GIBCO). The composition of the intrapipette solution was (mM) KCl, 135; $\mathrm{CaCl}_{2}, 0.5$; HEPES, 5; EGTA, 5; ATP-2Na, 5; GTP-3Na, 1; and the $\mathrm{pH}$ was adjusted to 7.3 
with $\mathrm{KOH}$. The resistance of patch pipettes was 5-13 $\mathrm{M} \Omega$ when filled with an intrapipette solution. The membrane current was recorded with a patch-clamp amplifier (Axopatch-200B; Axon Instruments, Foster City, CA, USA) at 500 or $2,000 \mathrm{~Hz}$ through a DigiData 1322A Interface using pCLAMP software (version 9.0, Axon Instruments).

\section{Morphological Analysis}

Experiments were carried out under non-blinded conditions. Infected fibroblasts were fixed by $0.1 \mathrm{M}$ phosphate buffered saline containing $4 \%$ paraformaldehyde for $5 \mathrm{~min}$. Photomicrographs of fixed cells were taken under a microscope (BZ-9000, Keyence, Osaka, Japan) equipped with software (BZ-II Viewer, Keyence). Morphological parameters were analyzed by a BZ-II analyzer (Keyence). The soma size was calculated as the area surrounded by the base of neurites.

\section{Physiological Analysis}

To assess photoresponses, we calculated the holding current and baseline noise before light stimulation for $>6$ s. When the holding current was larger than $-500 \mathrm{pA}$ or the standard deviation of baseline noise was larger than 7.0 pA, the data were excluded from the analysis. When the stepwise baseline shift started after light stimulation with a modest delay, we judged it as an artificial baseline shift and excluded it from the analysis. The photo stimulation-induced current around the maximum current amplitude was sampled for $1 \mathrm{~s}$ during the light stimulation. The peak amplitude was calculated as the mean of the sampled photo stimulation-induced current. A peak amplitude larger than twice that of the baseline noise (standard deviation of baseline) before photo stimulation was classified as a "photo response". Total charge transfer across the membrane during light stimulation was integrated over time and normalized to the charge transfer per second ${ }^{10}$.

\section{Statistical Analysis}

We used the statistical software "EZR"11. Morphological data were analyzed by the Kruskal-Wallis test. After this test, statistics for individual data were performed using the Steel-Dwass test. Electrophysiological data were analyzed by Fisher's exact test.

\section{Results}

Morphology of Induced Photosensitive Cells from Fibroblasts

To investigate the morphological changes in cells, first, we compared the basic morphological properties between fibroblast cell lines, Fib\#1 and Fib\#2. In non-infected fi- broblast cell lines, most cells were fusiform (Fig. 1A and C). The soma size of Fib\#1 was significantly larger than that of Fib\#2 (mean \pm SEM, Fib\#1: $961.5 \pm 48.8 \mu \mathrm{m}^{2}, \mathrm{n}=186$, Fib\#2: $509.2 \pm 28.3 \mu \mathrm{m}^{2}, \mathrm{n}=158, P<0.001$, Steel-Dwass test) (Fig. 1E). In contrast, no difference was found in the number of primary processes between the cell lines (mean \pm SEM, Fib\#1: 3.8 $\pm 0.1, \mathrm{n}=182$, Fib\#2: 3.5 $\pm 0.1, \mathrm{n}=158$, $P>0.05$, Steel-Dwass test).

In CRNO-infected fibroblast cell lines, we observed very large cells with multiple primary processes (neurites) among the CRNO-infected cells (Fig. 1B and D). The soma size and the number of processes varied among cells. In both cell lines, the soma size of CRNOinfected cells was significantly larger than the soma size of non-infected cells (mean \pm SEM, Fib\#1: 3,692.5 \pm $204.8 \mu^{2}, \mathrm{n}=205$ for cells and $\mathrm{n}=4$ for cover slips, $P<0.01$, Fib\#2: $4,989.6 \pm 324.8 \mu^{2}, n=137$ for cells and $n=9$ for cover slips, $P<0.001$ ) (Fig. 1E). We also observed a significant increase in the number of primary processes in CRNO-infected cell lines (mean \pm SEM, Fib\#1: $7.3 \pm 0.2, \mathrm{n}=$ 200 for cells and $\mathrm{n}=5$ for cover slips, $P<0.001$, Fib\#2: $8.0 \pm$ $0.3, n=136$ for cells and $n=7$ for cover slips, $P<0.001$ ) (Fig. 1F). These data indicated that $C R N O$ transfection induces changes in the morphological properties of fibroblast cell lines.

We also investigated whether the morphological properties of CRNO-infected fibroblasts differ between the two fibroblast cell lines. Contrary to the non-infected condition, the soma size of Fib\#1 was significantly smaller than that of Fib\#2, while no difference was found in the number of primary processes between infected cell lines.

Response Properties to Light Stimuli in Induced Photosensitive Cells

Next, we investigated whether physiological properties differ between cell lines of fibroblasts. In the present study, we considered cells with large sized soma as representative CRNO-infected cells and recorded the current from those large sized cells (Fig. 1B and D) as they responded to light stimuli ${ }^{4}$.

Light responses in retinal neurons are classified into photoreceptor-type responses and intrinsically photosensitive ganglion cell (ipRGC)-type responses ${ }^{12}$. Although both types of light responses are mediated by cation channels (the main permeable ion is $\mathrm{Na}^{+}$), their polarity is opposite. Light stimulation closes cation channels and hyperpolarizes photoreceptor-type cells, but opens cation channels and depolarizes ipRGC-type cells. In the present study, we set the holding potential close to the mem- 
A


B

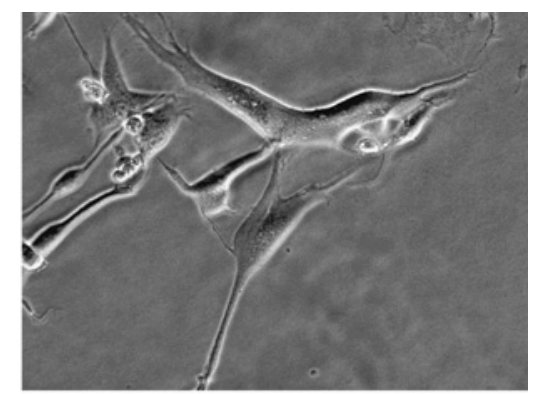

$\mathrm{D}$

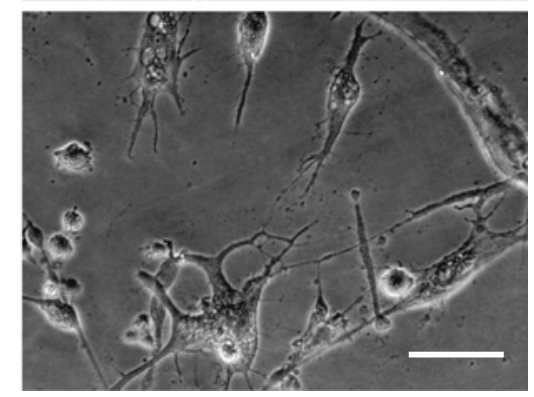

$\mathrm{F}$

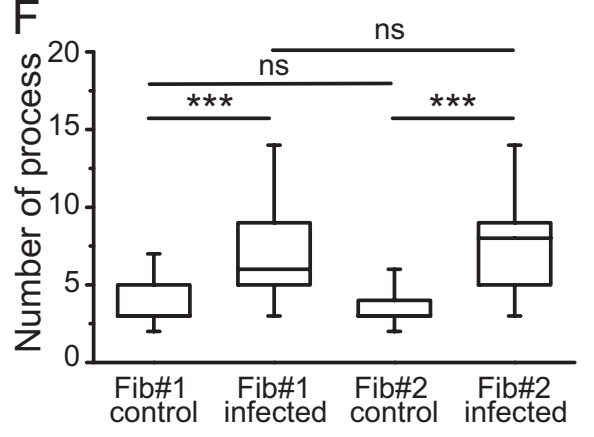

Fig. 1 Effects of $C R N O$-infection on the morphology of two fibroblast cell lines. A: noninfected Fib\#1 (Fib\#1 control), B: CRNO-infected Fib\#1 (Fib\#1 infected), C: non-infected Fib\#2 (Fib\#2 control), D: CRNO-infected Fib\#2 (Fib\#2 infected), bar: $50 \mu \mathrm{m}$, E and F: Statistical analysis of the soma size and the number of primary processes. Whiskers show the 5th and 95th percentiles, and box boundaries show the 25th and 75th percentiles. Horizontal lines correspond to the median. $* * * \mathrm{p}<0.001$, $* * \mathrm{p}<0.01$ n.s. not significant.

brane potential of photoreceptors in the dark $(-40 \mathrm{mV})^{13,14}$ to separate these two types of photoresponses. In our experimental condition, the closure of $\mathrm{Na}$ channels (photoreceptor-type response) is detected as an outward current, while the opening of $\mathrm{Na}$ channels (ipRGC-type response) is detected as an inward current (reversal potential of $\mathrm{Na}$ is $+60 \mathrm{mV}$ ).

A detectable current was observed in both Fib\#1 and Fib\#2 fibroblasts when photo-stimulation was found (Fig. 2A and C). We observed both an outward current and an inward current. The outward current started to flow immediately and continued to flow even after the cessation of light stimuli (Fig. 2A). The inward current also started to flow without detectable delay and increased in amplitude quickly, and then increased in amplitude gradually until the end of light stimulation (Fig. 2C). The current flow decreased gradually after the cessation of light stim- uli and eventually returned to the baseline level. Light stimulation did not produce any detectable fluctuation of the baseline in non-infected cells in either cell line (Fig. 2B and D). Since the distinct response to light was observed only once in most cells examined, we did not use the same cover slip for other trials.

To assess how many CRNO-infected cells responded to light, we calculated the peak amplitude and the total charge transferred across the membrane during light stimulation. Distinct "photo responses" were observed in 6 cells in CRNO-infected Fib\#1 fibroblasts $(n=20)$ and 17 cells in CRNO-infected Fib\#2 fibroblasts $(n=33)$ (Fig. 2E, open circle). Detectable "photo responses" were observed in no cells in the non-infected Fib\#1 fibroblasts $(n=15)$ and 1 cell in the non-infected Fib\#2 fibroblasts $(n=10)$ (Fig. 2E). The ratio of cells with "photo responses" was significantly greater in CRNO-infected cells than in non- 


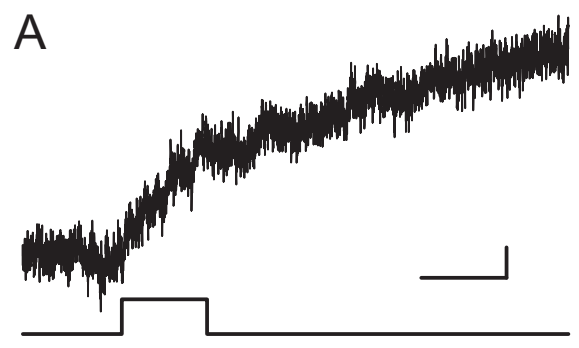

B
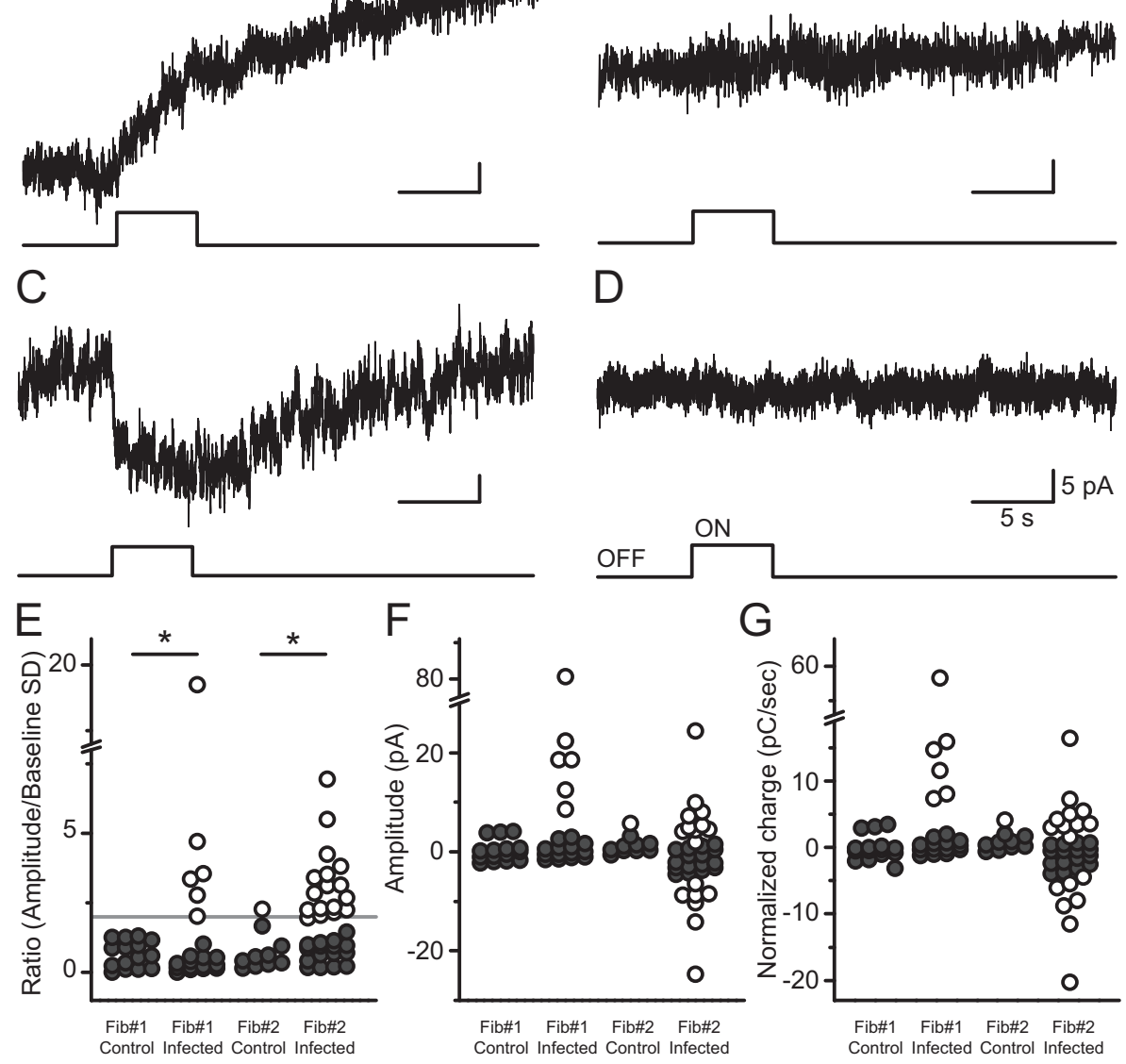

Fig. 2 The light responses in two CRNO-infected fibroblast cell lines. A and B: Responses to light in CRNO-infected (A) and the non-infected cells (B) in Fib\#1. C and D: Responses to light in CRNO-infected (C) and the non-infected cells (D) in Fib\#2. Timing and duration of light stimulation are shown under the current trace. The holding potential was $-40 \mathrm{mV}$. E: The relationship between the peak current amplitude to photo stimulation and the baseline noise level. The cells with "photo responses" are indicated by open circles. Solid line (drawn at 2 in the ordinate) indicates the threshold of "photo responses". * $<<0.05$. F and G: Summary of the polarity of the peak current amplitudes (F) and normalized charge-transfer (G) during photostimulation. Open circles and filled circles in F and G correspond to the open circles and the filled circles shown in E, respectively. Number of cover slips used for the experiments is equal to the number of samples.

infected cells in both cell lines $(P<0.05$, Fisher's exact test). However, the types of light responses in CRNOinfected cells were different between two cell lines. Only an outward current was observed in Fib\#1 fibroblasts, while both an inward current and an outward current were observed in Fib\#2 fibroblasts (Fig. 2F, open circle). The distribution of total charge-transfer of cells with "photo responses" was similar to the distribution of current amplitude (Fig. 2G, open circle), confirming the plausibility of our criteria.

We also assessed whether the "photo responses" lasted after the light stimulation. We measured the amplitude of "photo responses" at $30 \mathrm{~s}$ after the light stimulation in 20 cells ( 3 cells were excluded from the analysis because of the deterioration of the whole cell configuration after light stimulation). In 13 cells with the outward current (total of Fib\#1 and Fib\#2), 11 cells still satisfied the criteria for "photo responses". In 7 cells with the inward current (Fib\#2 only), 2 cells satisfied the criteria for "photo responses".

\section{Discussion}

We showed that the type of fibroblast cell line might affect the physiological properties of induced photosensitive cells infected with CRNO genes by direct reprogramming. We found that "photo responses" in Fib\#1 fibro- 
blasts were an outward current only, while "photo responses" in Fib\#2 fibroblasts were an inward or an outward current. We also found a difference in the morphological properties of the photosensitive cells between the two fibroblast cell lines. This is the first report to describe that induced photosensitive cells produced by direct reprogramming can express a different phenotype.

Photo responses should adapt during light stimulation and disappear immediately after the cessation of light stimulation in the mammalian retina ${ }^{14}$. However, the outward current (photoreceptor like responses) did not recover even when light stimulation was halted. In the photoreceptors, once the photo-transduction cascade is activated, an activated phosphodiesterase continues to hydrolyze cGMP, leading to the reduction of the intracellular cGMP concentration and the closure of cGMP-gated channels ${ }^{15}$.

If the outward current is mediated by a similar cascade of photo-responses in photoreceptor-like cells, a poor inactivation of the outward current after light stimulation might be explained by an insufficient recovery of the intracellular cGMP concentration. Although the possibility that excess degradation of cGMP by the prolonged strong flash (present study, $5 \mathrm{~s}$ in duration) might hamper the quick recovery observed in the primate retina $(10 \mathrm{~ms}$ in duration $)^{14}$ can not be ruled out, CRNO-infected cells probably lack the complete machinery to halt the light responses. Similarly, the recovery of the inward current (ipRGC-like responses) was partial and slow. The signal cascade in ipRGCs is thought to be mediated by an increase in the concentration of second messengers (inositol triphosphate and diacylglycerol) ${ }^{12}$. If the inward current is mediated by a similar cascade in ipRGC-like cells, the degradation of synthesized second messengers is likely to terminate the photo-responses. However, partial recovery of the inward current might reflect superimposition of the delayed activation of photoreceptor-like responses.

The functional difference between the two cell lines was not predictable from their morphology, although there was a difference in the soma size between them. As the retinal neurons have a distinct morphology and function, it is likely that their morphology has a strong relationship with the function of the retinal neurons. The difference in soma size of CRNO-infected cells might reflect the difference of the "photo responses".

In the present study, we observed that the physiological function of the induced photosensitive cells by direct reprogramming differed between two cell lines. Fib\#2 differentiated into either photoreceptor-like or ipRGC-like cells, while Fib\#1 differentiated into only photoreceptorlike cells. The present results corresponded well with previous findings in the differentiated cells derived from ES or iPS cells ${ }^{6-9}$, suggesting that the source of the cell can determine the fate of the differentiated cells in the direct reprogramming method. In the cardiomyocytes derived from ES and iPS cell lines, it is difficult to clearly predict how many functional cardiomyocytes are formed based on the simple expression of molecular markers, although the higher expression level of molecular markers was detected in the well-differentiated line $e^{6,9}$, suggesting that the variation of the induced cell types with different physiological properties reflects the difference of the intrinsic properties between Fib\#1 and Fib\#2. In the retinal pigment epithelium, the handling during culture (passage number, initial cell seeding density, composition of culture medium etc.) also determines the yield of the differentiated cells ${ }^{8}$. Although we have handled the two cell lines with the same protocol, we have to be careful about the possibility that the physiological properties of the differentiated cells might be affected by the culture condition. It will be interesting to see if there are additional factors that affect the physiological properties of the differentiated cells in future studies.

Acknowledgements: We would like to express our sincere thanks to Kiyoko Miyamoto and Yukari Komuta for their technical assistance during this work. We are grateful to the National Eye Institute, National Institutes of Health for the gift of 11-cis retinal.

This work was supported by a Grant-in-Aid for Young Scientists (B) from JSPS (KAKENHI No. 26860150 and 17K16990) to T. Ishii, Novartis Pharma Research Grants 2017 to T. Ishii, a Grant-in-Aid for challenging Exploratory Research (KAKENHI No. 25670741) to Y. Seko, Grants from the Japan Society for the Promotion of Science (Grant-in-Aid for Scientific Research 15H04998) to Y. Seko, Grants of the National Rehabilitation Center for Persons with Disabilities to Y. Seko, a Nippon Medical School Grant-in-Aid for Medical Research to M. Kaneda and Novartis Pharma Research Grants to M. Kaneda.

Conflict of Interest: The authors have no actual or potential conflicts of interest, including any financial, personal, or other relationships with people or organizations that could influence the present article.

Role of authors: TI planned the experimental design, conducted the electrophysiological and morphological studies, 
and participated in the writing of the manuscript. CY conducted the morphological study. YS conducted the preparation of reprogrammed cells and participated in the writing of the manuscript. AU provided the materials and participated in the writing of the manuscript. MK planned the experimental design, conducted the electrophysiological study, and participated in the writing of the manuscript.

\section{References}

1. Osakada F, Ikeda H, Mandai M, Wataya T, Watanabe $\mathrm{K}$, Yoshimura N, Akaike A, Sasai Y, Takahashi M: Toward the generation of rod and cone photoreceptors from mouse, monkey and human embryonic stem cells. Nat Biotechnol 2008; 26: 215-224.

2. Eiraku M, Takata N, Ishibashi H, Kawada M, Sakakura E, Okuda S, Sekiguchi K, Adachi T, Sasai Y: Self-organizing optic-cup morphogenesis in three-dimensional culture. Nature 2011; 472: 51-56.

3. Schwartz S, Hubschman J, Heilwell G, Franco-Cardenas V, Pan C, Ostrick R, Mickunas E, Gay R, Klimanskaya I, Lanza R: Embryonic stem cell trials for macular degeneration: a preliminary report. Lancet 2012; 379: 713-720.

4. Seko Y, Azuma N, Ishii T, Komuta Y, Miyamoto K, Miyagawa $\mathrm{Y}$, Kaneda M, Umezawa A: Derivation of human differential photoreceptor cells from adult human dermal fibroblasts by defined combinations of CRX, RAX, OTX2 and NEUROD. Genes Cells 2014; 19: 198-208.

5. Seko Y, Azuma N, Kaneda M, Nakatani K, Miyagawa Y, Noshiro Y, Kurokawa R, Okano H, Umezawa A: Derivation of human differential photoreceptor-like cells from the iris by defined combinations of CRX, RX and NEUROD. PLoS One 2012; 7: e35611.

6. Kaichi S, Hasegawa K, Takaya T, Yokoo N, Mima T, Kawamura T, Morimoto T, Ono K, Baba S, Doi H, Yamanaka S, Nakahata T, Heike T: Cell line-dependent differentiation of induced pluripotent stem cells into cardiomyocytes in mice. Cardiovasc Res 2010; 88: 314-323.
7. Lamba DA, McUsic A, Hirata RK, Wang PR, Russell D, Reh TA: Generation, purification and transplantation of photoreceptors derived from human induced pluripotent stem cells. PLoS One 2010; 5: e8763.

8. Lane A, Philip LR, Ruban L, Fynes K, Smart M, Carr A, Mason C, Coffey P: Engineering efficient retinal pigment epithelium differentiation from human pluripotent stem cells. Stem Cells Transl Med 2014; 3: 1295-1304.

9. Pekkanen-Mattila M, Kerkela E, Tanskanen JM, Pietila M, Pelto-Huikko M, Hyttinen J, Skottman H, Suuronen R, Aalto-Setala K: Substantial variation in the cardiac differentiation of human embryonic stem cell lines derived and propagated under the same conditions--a comparison of multiple cell lines. Ann Med 2009; 41: 360-370.

10. Komuta $\mathrm{Y}$, Ishii T, Kaneda M, Ueda $\mathrm{Y}$, Miyamoto $\mathrm{K}$, Toyoda M, Umezawa A, Seko Y: In vitro transdifferentiation of human peripheral blood mononuclear cells to photoreceptor-like cells. Biology open 2016; 5: 709-719.

11. Kanda Y: Investigation of the freely available easy-to-use software 'EZR' for medical statistics. Bone marrow transplantation 2013; 48: 452-458.

12. Hankins MW, Peirson SN, Foster RG: Melanopsin: an exciting photopigment. Trends Neurosci 2008; 31: 27-36.

13. Kaneko A, Tachibana M: Membrane properties of solitary retinal cells. In Progress in Retinal Research Vol. 5 (Osborne NaC J, ed), 1986; pp 125-146, Pergamon Press, Oxford and New York.

14. Schneeweis DM, Schnapf JL: Photovoltage of rods and cones in the macaque retina. Science 1995; 268: 1053-1056.

15. Torre V, Matthews HR, Lamb TD: Role of calcium in regulating the cyclic GMP cascade of phototransduction in retinal rods. Proceedings of the National Academy of Sciences of the United States of America 1986; 83: 71097113.

(Received, November 24, 2017)

(Accepted, December 25, 2017) 